\title{
Influencing factors of unmet needs for child spacing among selected countries experiencing high maternal-mortality in sub-Saharan Africa
}

\author{
Abayomi T. Olarinmoye ${ }^{1}$, Olanrewaju D. Eniade ${ }^{2,3 *}$, Olariike O. Kayode ${ }^{1}$, \\ Abimbola A. Abiola ${ }^{1}$, Modupe O. Onifade ${ }^{1}$, Sarafa O. Odewale ${ }^{1}$, Bode Kayode ${ }^{1}$
}

\begin{abstract}
${ }^{1}$ Department of Public Health, Faculty of Basic Medical Sciences, Adeleke University Ede, Osun State. Nigeria ${ }^{2}$ Department of International Research Centre for Excellence, Institute of Human Virology, Nigeria

${ }^{3}$ Department of Epidemiology and Medical Statistics, University of Ibadan, Nigeria
\end{abstract}

Received: 11 September 2021

Revised: 15 October 2021

Accepted: 16 October 2021

\section{*Correspondence:}

Dr. Olanrewaju D. Eniade,

E-mail: eniadetreasure@gmail.com

Copyright: () the author(s), publisher and licensee Medip Academy. This is an open-access article distributed under the terms of the Creative Commons Attribution Non-Commercial License, which permits unrestricted non-commercial use, distribution, and reproduction in any medium, provided the original work is properly cited.

\section{ABSTRACT}

Background: Maternal complication and death have implicated unintended pregnancy. The indicator for measuring the risk of unintended pregnancy is unmet need for family planning (FP). It is necessary to explore the current situation of unmet need for child spacing (UNCS) as well as its influencing factors among countries that are experiencing high maternal mortality ratio. We aimed to unveil the prevalence of UNCS and its associated factors in Nigeria, Liberia and Sierra Leone.

Methods: We analysed the data from DHS phase VII survey, a cross- sectional study conducted from year 2017 to 2018 across several countries. Total records of 25,539, 5,553, and 10,050 were extracted for Nigeria, Liberia and Sierra Leone respectively. Explored variables were UNCS, demographic characteristics and husband's partners profile. Data was analysed using SPSS version 25. Descriptive statistics, test of association (chi-square) and binary logistic regression were used during the data analysis $\left(\alpha_{0.05}\right)$.

Results: UNCS was high in Nigeria (15.9\%), Liberia (22.5\%) and Sierra Leone (21.9\%). In Nigeria, Liberia and Sierra Leone, $40 \%, 37 \%$ and $49.1 \%$ were uneducated respectively. The proportion of married women were $89 \%$ in Nigeria, $32 \%$ in Liberia and $70.2 \%$ in Sierra Leone. Also, female headship of household (OR: 1.29, 95\%CI: 1.09-1.54) was associated with UNCS relative to male headship of household.

Conclusions: UNCS was high in the three countries. Factors like educational status of women, age, as well as women as head of the households should be given much attention in the efforts to reduce UNCS as identified in this study.

Keywords: Family planning, Maternal mortality, Unmet need for child spacing

\section{INTRODUCTION}

Spurred by the 2030 sustainable development goals of the United Nations towards the betterment of the global sexual and reproductive health. The 2030 SDG was to reduce maternal mortality to 70 per 100,000 life birth worldwide. ${ }^{1}$ The $3^{\text {rd }}$ goal on the list states that healthy lives and promotion of well-being for all at all ages should be ensured by $2030 .{ }^{1}$ As the efforts to achieve these goals are ongoing in most of the developed countries and even in some of the developing countries, it is necessary to explore the surrounding factors of maternal mortality. Unintended pregnancy leads to abortion, medical complications and psychological issues which are major causes of maternal complication such as pre-eclampsia, postpartum hemorrhage, postpartum pre-eclampsia, maternal depression and parenting stress. ${ }^{2}$ Unintended pregnancies was described as pregnancies that were reported to have been either unwanted (no children or no more children were desired) or mistimed (occurred earlier than desired). ${ }^{3}$ Further, unintended pregnancies being a major cause of 
maternal complications and mortality have also implicated unmet need for contraception. ${ }^{4}$

The United Nations defined unmet needs for family planning (FP) as in-union pregnant women whose pregnancies were unwanted or mistimed at the time of conception, in-union postpartum amenorrheic women who were not on contraceptives and whose last birth was unwanted or mistimed, and all in-union fecund women who were neither pregnant nor postpartum amenorrheic, and who either want to limit family size or want to space births, but were not on any contraceptives. ${ }^{5}$ The demographic and health survey also defined unmet need for family planning as the percentage of women who do not want to become pregnant but are not using contraception. ${ }^{6}$ Also, the World Health Organization (WHO) described women with unmet need as those who are fecund and sexually active but are not using any method of contraception, and report not wanting any more children or wanting to delay the next child. The WHO further explained that the concept of unmet need is to identify the gap between women's reproductive intentions and their contraceptive behavior.?

Globally, maternal mortality ratio has decreased from 342 deaths per 100,000 in year 2000 to 211 deaths per 100,000 in year $2017 .{ }^{8}$ But unfortunately still high in the African countries as $86 \%$ of the maternal death that occurred worldwide was contributed by sub-Saharan African countries. ${ }^{8}$ About $8 \%$ of the maternal death that occurred worldwide was traced to abortion which was as a result of unintended pregnancy, other causes were preventable maternal complications. ${ }^{8}$ Among the sub-Sahara African countries, Nigeria (512 per 100,000), Sierra Leone (717per 100,000) and Liberia (742 per 100,000$)$ witnessed the highest maternal mortality ratio. ${ }^{9}$ Also, unmet need for FP was reported to be on the high side in the three countries, $33.4 \%$ in Liberia, $18.9 \%$ in Nigeria and $24.8 \%$ in Sierra Leone. ${ }^{9}$ Since maternal mortality has been traced to unmet needs for FP (child limiting and spacing) through unintended pregnancy, it has become essential to do enough exploration on unmet need for child spacing and child limiting among the three sub-Sahara African countries (Nigeria, Liberia and Sierra Leone).

Unmet need for FP is a major public health concern globally, for instance in $2017,12 \%$ of married or in-union women are estimated to have an unmet need for family planning. ${ }^{10}$ Also, African countries were reported to be the highest contributor of unmet need for FP with the prevalence $22 \% .{ }^{10}$ However, the concern of high prevalence of unmet need for FP have received meaningful attention by scholars, leading to reduction in the prevalence of unmet need for FP in the developed countries. ${ }^{11}$ But the prevalence of unmet need for FP is still high in some sub-Sahara African countries (such as Nigeria, Liberia and Sierra Leone), Most studies focused on the general unmet need for FP, and limited information was provided on unmet need for child spacing despite the fact of its harm towards maternal complication and maternal death. ${ }^{2}$ Also, there are paucity of information on the role of demographic attributes, socio-economic characteristics and partner profile on unmet need for child spacing in the three selected countries (Nigeria, Liberia and Sierra Leone). Therefore, this study explored the prevalence of unmet need for child spacing and its associated factors in the three selected countries.

\section{METHODS}

A cross-sectional study was conducted from year 2017 to 2018 across several countries. We analysed the data from Demographic and Health Survey (DHS) phase VII survey which was the DHS survey collected data that are comparable across countries, further details of this survey is available in the DHS report. ${ }^{12}$ A stratified sample was selected in two stages by separating each states into urban and rural areas, further details was provided in the DHS report $2018 .^{12,13}$

Sub-Saharan countries that have high maternal mortality rate DHS phase-VII survey were selected for this analysis namely Nigeria, Liberia and Sierra Leone. Women's recode (IR) consisting the records for women aged 15 to 49 years were extracted from the phase-7 2018 DHS dataset for the three selected countries. The explanatory variables that were explored in this study are; age, highest level of education, respondents and partner occupation status, marital status, media use (watching of television, listening to radio, reading of newspaper of magazine), number of children, wealth index, sex of head of the household, place of residence, age at first sex, decision maker for not using contraceptive, remarriage status, living together with husband, partner's level of education, and number of wives the husband has.

After deleting records with missing data for unmet need in the individual recode dataset, 25,539 records was extracted for Nigeria, 5,553 records for Liberia, and 10,050 for Sierra Leone. Details for sample size calculation in DHS can be found in the DHS report. ${ }^{14}$

\section{Data analysis}

The dataset was sorted by the outcome variable (unmet need for child spacing). Descriptive analysis was done to assess the prevalence of unmet need for child spacing and all other explanatory variables were also analysed using the frequency and percentage distribution for the categorical variables, while mean and standard deviation were used for the quantitative variables. Association with unmet need for child spacing were examined using chisquare test, for the categorical explanatory variables versus unmet need for child spacing, while independent t-test was used for the quantitative explanatory variables versus unmet need for child spacing. After the test of association, variables that were significantly associated with unmet need for child spacing at $5 \%$ level of significance were step wisely included into logistic regression model. Three models were fitted. Model-I was fitted to explore factors 
associated with unmet need for child spacing in Nigeria. Model-II was fitted to explore factors associated with unmet need for child spacing in Liberia. Model-III was fitted to explore factors associated with unmet need for child spacing in Sierra Leone.

\section{Ethical consideration}

We made use of secondary data for this study and ethical approval was obtained for the primary data collection by the DHS team. Every confidential variables and personal identifier have been excluded from the dataset before it was released to us for this study. As a result, the confidentiality and anonymity of the respondents are guaranteed. Also, permission to use the DHS dataset was requested and granted by DHS.

\section{RESULTS}

\section{Socio-demographic characteristics of respondents in Nigeria}

The prevalence of unmet need for child spacing was shown in Figure 1, and profile of the respondents and their partners were presented in Table 1. Unmet need for child spacing was high in Nigeria (15.9\%). About 8.6\% were aged 15-19 years, $40 \%$ were uneducated, lower proportion $(10.3 \%)$ had higher education. Also, $68.3 \%$ were currently working and $88.8 \%$ were married. This study further revealed that $88.5 \%$ were using media (watching television, listening to radio and reading newspaper/ magazine). Majority (60.5\%) of the respondents had one or two children. The wealth index showed that $42.1 \%$ were poor. Lower proportion $(11.3 \%)$ of the households were headed by female, and $37.2 \%$ reside in the urban area. About $42.3 \%$ of the respondents reported that decision for not taking up contraceptive was jointly taken with their partners. Also, 414 (1.7\%) of the respondents remarried and $9.1 \%$ were not living with their partners. The study participants constituted $45.0 \%$ Christians and $54.3 \%$ Muslims. Higher proportion (19.2\%) of the respondent's partners were aged $\geq 50$ years, majority (35.3\%) have secondary education, $28.2 \%$ were married to more than one wife, and $3.6 \%$ of the partners were not working.

\section{Socio-demographic characteristics of respondents in Liberia}

The prevalence of unmet need for child spacing was shown in Figure 1, and profile of the respondents and their partners were presented in Table 1. Unmet need for child spacing was as well high in Liberia (22.5\%). Age group 20 -24 years had the highest proportion $(20.6 \%), 37.0 \%$ of the respondents were uneducated and $34 \%$ were not working. Also, 32\% were married, 94\% were using media (watching television, listening to radio and reading news/magazine). The wealth index showed that $53.1 \%$ poor. Household headed by women were about $33.2 \%$ and a higher proportion $(59.9 \%)$ live in the rural areas. The mean age at first sexual intercourse was $18.4 \pm 3.55$ years. About $33.9 \%$ of the respondents reported that decision for not taking up contraceptive was jointly taken with their partners, $8.2 \%$ have remarried and majority $(98.2 \%)$ of the respondents were Christians. Further, the proportion of partners' age group ranged from $6.5 \%$ (less than 25years) to $20.5 \%$ (3539 years). About $26.9 \%$ of their partners were uneducated while most $(43.1 \%)$ had secondary education. We observed that $565(11.4 \%)$ had more than one wife and $8.3 \%$ were not working.

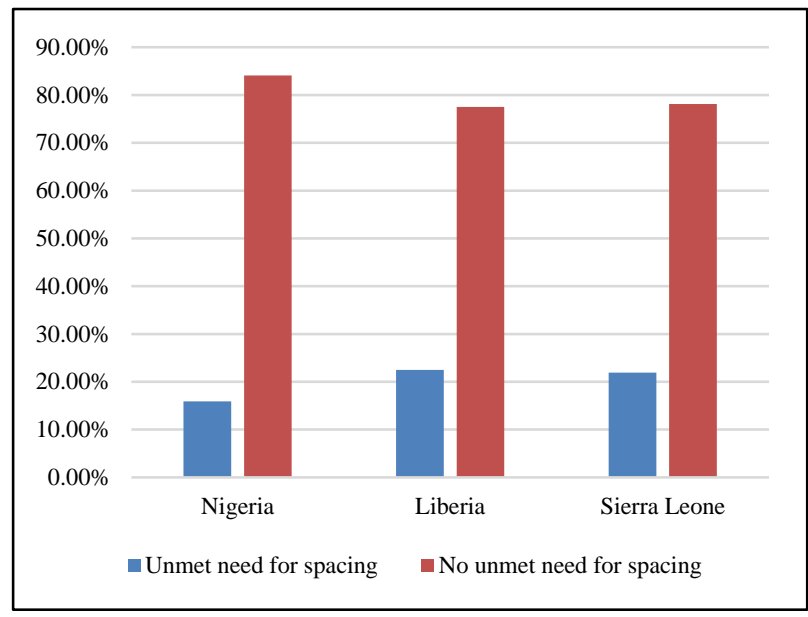

Figure 1: Bar chart showing unmet need for child spacing in Nigeria, Liberia and Sierra Leone (\%).

\section{Socio-demographic characteristics of respondents in Sierra Leone}

The prevalence of unmet need for child spacing was shown in Figure 1, and profile of the respondents and their partners were presented in Table 1. Unmet need for child spacing was also high in Sierra Leone (21.9\%). Higher proportion (14.4\%) of age group 15-19 years. A lot (49.1\%) were uneducated and $25.5 \%$ were not working. Also, $70.2 \%$ were currently married and $95.6 \%$ exposed to media (watching television, listening to radio and reading newspaper/magazine). Higher proportion $(77.4 \%$ ) had $>2$ children. The wealth index showed that $40.7 \%$ were poor, $20.6 \%$ were averagely wealthy while $38.6 \%$ were rich. Households headed by women were about $25.4 \%$ and a higher proportion $(60.7 \%)$ live in the rural area. The mean age at first sexual intercourse was $19 \pm 3.99$ years. About $26.5 \%$ of the respondents reported that decision for not taking up contraceptive was jointly taken with their partners. We observed that $5.3 \%$ had remarriage and $19 \%$ were not staying with their husbands/partners. Also, all the respondents were Christians. Further, partners' age group distribution ranged from $4.2 \%$ (less than 25 years) to $20.7 \%$ (35-39 years). About $54.5 \%$ of their partners were uneducated. We observed that $28.6 \%$ had more than one wife and $5.3 \%$ were not working. 
Table 1: Socio-demographic characteristics of respondents in Nigeria, Liberia and Sierra Leone.

\begin{tabular}{|c|c|c|c|c|c|c|}
\hline \multirow{2}{*}{ Variables } & \multicolumn{2}{|l|}{ Nigeria } & \multicolumn{2}{|l|}{ Liberia } & \multicolumn{2}{|c|}{ Sierra Leone } \\
\hline & N (25539) & $\%$ & $\mathbf{N}(\mathbf{5 5 5 3})$ & $\%$ & $\mathbf{N}(\mathbf{1 0 0 5 0})$ & $\%$ \\
\hline \multicolumn{7}{|l|}{ Age in year } \\
\hline $15-19$ & 2189 & 8.6 & 845 & 15.2 & 1448 & 14.4 \\
\hline $20-24$ & 4806 & 18.8 & 1142 & 20.6 & 2071 & 20.6 \\
\hline $25-29$ & 5975 & 23.4 & 1014 & 18.3 & 2249 & 22.4 \\
\hline $30-34$ & 4963 & 19.4 & 882 & 15.9 & 1630 & 16.2 \\
\hline $35-39$ & 4103 & 16.1 & 877 & 15.8 & 1631 & 16.2 \\
\hline $40-44$ & 2316 & 9.1 & 540 & 9.7 & 672 & 6.7 \\
\hline $45-49$ & 1187 & 4.6 & 253 & 4.6 & 349 & 3.5 \\
\hline \multicolumn{7}{|l|}{ Highest educational level } \\
\hline No education & 10220 & 40.0 & 2052 & 37.0 & 4930 & 49.1 \\
\hline Primary & 3915 & 15.3 & 1600 & 28.8 & 1294 & 12.9 \\
\hline Secondary & 8768 & 34.3 & 1735 & 31.2 & 3425 & 34.1 \\
\hline Higher & 2636 & 10.3 & 166 & 3.0 & 401 & 4.0 \\
\hline \multicolumn{7}{|l|}{ Respondent currently working } \\
\hline No & 8089 & 31.7 & 1890 & 34.0 & 2564 & 25.5 \\
\hline Yes & 17450 & 68.3 & 3663 & 66.0 & 7486 & 74.5 \\
\hline \multicolumn{7}{|l|}{ Current marital status } \\
\hline Never in union & 1644 & 6.4 & 1308 & 23.6 & 2246 & 22.3 \\
\hline Married & 22680 & 88.8 & 1779 & 32.0 & 7056 & 70.2 \\
\hline Living with partner & 859 & 3.4 & 2100 & 37.8 & 486 & 4.8 \\
\hline Widowed & 123 & 0.5 & 37 & 0.7 & 71 & 0.7 \\
\hline Divorced & 74 & 0.3 & 25 & 0.5 & 27 & 0.3 \\
\hline No longer living together/separated & 159 & 0.6 & 304 & 5.5 & 164 & 1.6 \\
\hline \multicolumn{7}{|l|}{ Media use } \\
\hline No & 2930 & 11.5 & 333 & 6.0 & 444 & 4.4 \\
\hline Yes & 22609 & 88.5 & 5220 & 94.0 & 9606 & 95.6 \\
\hline \multicolumn{7}{|c|}{ Number of children 5 and under in household } \\
\hline$\leq 2$ & 4711 & 18.4 & 1399 & 25.2 & 2272 & 22.6 \\
\hline$>2$ & 20828 & 81.6 & 5154 & 74.8 & 7778 & 77.4 \\
\hline \multicolumn{7}{|l|}{ Wealth index } \\
\hline Poor & 10740 & 42.1 & 2947 & 53.1 & 4095 & 40.7 \\
\hline Average & 5191 & 20.3 & 1176 & 21.2 & 2073 & 20.6 \\
\hline Rich & 9608 & 37.6 & 1430 & 25.8 & 3882 & 38.6 \\
\hline \multicolumn{7}{|l|}{ Sex of household head } \\
\hline Male & 22643 & 88.7 & 3709 & 66.8 & 7502 & 74.6 \\
\hline Female & 2896 & 11.3 & 1844 & 33.2 & 2548 & 25.4 \\
\hline \multicolumn{7}{|l|}{ Type of place of residence } \\
\hline Urban & 9500 & 37.2 & 2224 & 40.1 & 3946 & 39.3 \\
\hline Rural & 16039 & 62.8 & 3329 & 59.9 & 6104 & 60.7 \\
\hline \multicolumn{7}{|l|}{ Remarriage } \\
\hline No & 24037 & 98.3 & 4739 & 91.8 & 8369 & 94.7 \\
\hline Remarriage & 414 & 1.7 & 426 & 8.2 & 464 & 5.3 \\
\hline \multicolumn{7}{|l|}{ Religion } \\
\hline Christians & 11503 & 45.0 & 5453 & 98.2 & 10048 & 100.0 \\
\hline Islam & 13869 & 54.3 & 32 & 0.6 & & \\
\hline Others & 167 & 0.7 & 68 & 1.2 & 2 & 0.0 \\
\hline \multicolumn{7}{|l|}{ Husband/partner's age } \\
\hline less than 25 & 591 & 2.5 & 254 & 6.5 & 313 & 4.2 \\
\hline $25-29$ & 2267 & 9.6 & 552 & 14.2 & 866 & 11.5 \\
\hline $30-34$ & 4042 & 17.2 & 671 & 17.3 & 1212 & 16.1 \\
\hline $35-39$ & 4651 & 19.8 & 796 & 20.5 & 1562 & 20.7 \\
\hline $40-44$ & 4176 & 17.7 & 683 & 17.6 & 1215 & 16.1 \\
\hline $45-49$ & 3286 & 14.0 & 491 & 12.7 & 1082 & 14.3 \\
\hline 50 or more & 4526 & 19.2 & 432 & 11.1 & 1292 & 17.1 \\
\hline
\end{tabular}

Continued. 


\begin{tabular}{|c|c|c|c|c|c|c|}
\hline \multirow{2}{*}{ Variables } & \multicolumn{2}{|l|}{ Nigeria } & \multicolumn{2}{|l|}{ Liberia } & \multicolumn{2}{|c|}{ Sierra Leone } \\
\hline & N (25539) & $\%$ & $\mathbf{N}(\mathbf{5 5 5 3})$ & $\%$ & N (10050) & $\%$ \\
\hline \multicolumn{7}{|c|}{ Husband/partner's education level } \\
\hline No education & 7772 & 33.0 & 1045 & 26.9 & 4114 & 54.5 \\
\hline Primary & 3400 & 14.4 & 614 & 15.8 & 584 & 7.7 \\
\hline Secondary & 8300 & 35.3 & 1673 & 43.1 & 1972 & 26.1 \\
\hline Higher & 3729 & 15.8 & 294 & 7.6 & 646 & 8.6 \\
\hline Don't know & 338 & 1.4 & 253 & 6.5 & 226 & 3.0 \\
\hline \multicolumn{7}{|l|}{ Number of other wives } \\
\hline 1 & 16821 & 71.5 & 3368 & 86.8 & 5375 & 71.3 \\
\hline$>1$ & 6640 & 28.2 & 444 & 11.4 & 2154 & 28.6 \\
\hline Don't know & 78 & 0.3 & 67 & 1.7 & 13 & 0.2 \\
\hline \multicolumn{7}{|c|}{ Husband/partner's occupation } \\
\hline Not working & 839 & 3.6 & 314 & 8.1 & 399 & 5.3 \\
\hline Working & 22700 & 96.4 & 3561 & 91.8 & 7143 & 94.7 \\
\hline \multicolumn{7}{|c|}{ Decision maker for not using contraception } \\
\hline Mainly respondent & 5488 & 37.3 & 1053 & 51.9 & 2391 & 52.4 \\
\hline Mainly husband, partner & 3004 & 20.4 & 288 & 14.2 & 961 & 21.1 \\
\hline Joint decision & 6222 & 42.3 & 687 & 33.9 & 1207 & 26.5 \\
\hline
\end{tabular}

\section{Factors independently associated with unmet need for child spacing in Nigeria}

As presented in Table 2, higher proportion of respondents aged $15-19$ years $(19.5 \%)$ and $20-24$ years $(19.6 \%)$ had unmet need for child spacing compared to respondents aged 45-49 years $(\mathrm{p}=0.000)$. More $(19.4 \%)$ of respondents who have attained secondary level of education had unmet need for child spacing in relative to $13.3 \%$ of uneducated who had unmet need for child spacing $(\mathrm{p}=0.000)$. Women who are never in a union had higher $(39.5 \%)$ unmet need for child compared to married women $(13.9 \%)(\mathrm{p}=0.000)$. Surprisingly, a higher percentage (17\%) of rich women had unmet need for child spacing compared to poor women $(14.4 \%)(\mathrm{p}=0.000)$. Also, unmet need for child spacing was higher $(22.6 \%)$ in the households headed by females $(\mathrm{p}=0.000)$. Further, number of five/under five children in the household $(\mathrm{p}=0.000)$, remarriage $(\mathrm{p}=0.000)$, cohabiting with husbands/partners $(\mathrm{p}=0.000)$, religion $(\mathrm{p}=0.000), \quad$ husbands/partners age $\quad(\mathrm{p}=0.000)$, husbands/partners level of education $(\mathrm{p}=0.000)$ and husband/partners occupation $(\mathrm{p}=0.015)$ were associated with unmet need for child spacing.

Table 2: Factors independently associated with unmet need for child spacing in Nigeria, Liberia and Sierra Leone.

\begin{tabular}{|c|c|c|c|c|c|c|c|c|c|}
\hline Variables & $\begin{array}{l}\text { Nigeria } \\
\text { Unmet need }\end{array}$ & $\left(\chi^{2}\right)$ & P value & $\begin{array}{l}\text { Liberia } \\
\text { Unmet need }\end{array}$ & $\left(\chi^{2}\right)$ & $P$ value & $\begin{array}{l}\text { Sierra Leone } \\
\text { Unmet need }\end{array}$ & $\left(\chi^{2}\right)$ & $P$ value \\
\hline Age in 5-year groups & & 217.72 & 0.000 & & 183.75 & 0.000 & & 102.85 & 0.000 \\
\hline $15-19$ & $427(19.5)$ & & & $300(35.5)$ & & & $379(26.2)$ & & \\
\hline $20-24$ & $940(19.6)$ & & & $303(26.5)$ & & & $519(25.1)$ & & \\
\hline $25-29$ & $1006(16.8)$ & & & $250(24.7)$ & & & $530(23.6)$ & & \\
\hline $30-34$ & $809(16.3)$ & & & $164(18.6)$ & & & $349(21.4)$ & & \\
\hline $35-39$ & $561(13.7)$ & & & $143(16.3)$ & & & $304(18.6)$ & & \\
\hline $40-44$ & $211(9.1)$ & & & $68(12.6)$ & & & $86(12.8)$ & & \\
\hline $45-49$ & $101(8.5)$ & & & $20(7.9)$ & & & $35(10.0)$ & & \\
\hline Highest educational lev & & 137.50 & 0.000 & & 41.40 & 0.000 & & 5.40 & 0.145 \\
\hline No education & $1356(13.3)$ & & & $380(18.5)$ & & & $1045(21.2)$ & & \\
\hline Primary & $597(15.2)$ & & & $400(25.0)$ & & & $295(22.8)$ & & \\
\hline Secondary & $1704(19.4)$ & & & $445(25.6)$ & & & $784(22.9)$ & & \\
\hline Higher & $398(15.1)$ & & & $23(13.9)$ & & & $78(19.5)$ & & \\
\hline Current work status & & 1.09 & 0.297 & & 22.71 & 0.000 & & 27.17 & 0.000 \\
\hline No & $1256(15.5)$ & & & $495(26.2)$ & & & $656(25.6)$ & & \\
\hline Yes & $2799(16.0)$ & & & $753(20.6)$ & & & $1546(20.7)$ & & \\
\hline Current marital status & & 828.59 & 0.000 & & 52.02 & 0.000 & & 39.00 & 0.000 \\
\hline Never in union & $649(39.5)$ & & & $339(25.9)$ & & & $559(24.9)$ & & \\
\hline Married & $3145(13.9)$ & & & $328(18.4)$ & & & $1487(21.1)$ & & \\
\hline
\end{tabular}




\begin{tabular}{|c|c|c|c|c|c|c|c|c|c|}
\hline Variables & $\begin{array}{l}\text { Nigeria } \\
\text { Unmet need }\end{array}$ & $\left(\chi^{2}\right)$ & P value & $\begin{array}{l}\text { Liberia } \\
\text { Unmet need }\end{array}$ & $\left(\chi^{2}\right)$ & $P$ value & $\begin{array}{l}\text { Sierra Leone } \\
\text { Unmet need }\end{array}$ & $\left(\chi^{2}\right)$ & P value \\
\hline Living with partner & $215(25.0)$ & & & $530(25.2)$ & & & $126(25.9)$ & & \\
\hline Widowed & $3(2.4)$ & & & $7(18.9)$ & & & $3(4.2)$ & & \\
\hline Divorced & $18(24.3)$ & & & $1(4.0)$ & & & $3(11.1)$ & & \\
\hline $\begin{array}{l}\text { No longer living } \\
\text { together/separated }\end{array}$ & $25(15.7)$ & & & $43(14.1)$ & & & $24(14.6)$ & & \\
\hline Media use & & 0.07 & 0.797 & & 0.70 & 0.404 & & 1.89 & 0.169 \\
\hline No & $470(16.0)$ & & & $81(24.3)$ & & & $109(24.5)$ & & \\
\hline Yes & $3585(15.9)$ & & & $1167(22.4)$ & & & $2093(21.8)$ & & \\
\hline \multicolumn{2}{|c|}{ Number of children $\leq 5$ year old } & 25.63 & 0.000 & & 12.73 & 0.005 & & 49.35 & 0.000 \\
\hline$\leq 2$ & $642(13.6)$ & & & $275(19.7)$ & & & $391(17.2)$ & & \\
\hline$>2$ & $2492(16.1)$ & & & $845(23.1)$ & & & $1484(22.9)$ & & \\
\hline Wealth index & & 31.02 & 0.000 & & 3.55 & 0.169 & & 8.32 & 0.016 \\
\hline Poor & $1545(14.4)$ & & & $645(21.9)$ & & & $874(21.3)$ & & \\
\hline Average & $873(16.8)$ & & & $256(21.8)$ & & & $422(20.4)$ & & \\
\hline Rich & $1637(17.0)$ & & & $347(24.3)$ & & & $906(23.3)$ & & \\
\hline \multicolumn{2}{|c|}{ Sex of household head } & 109.95 & 0.000 & & 0.51 & 0.477 & & 1.67 & 0.197 \\
\hline Male & $3401(15.0)$ & & & $844(22.8)$ & & & $1667(22.2)$ & & \\
\hline Female & $654(22.6)$ & & & $404(21.9)$ & & & $535(21.0)$ & & \\
\hline \multicolumn{2}{|c|}{ Type of place of residence } & 2.85 & 0.092 & & 1.13 & 0.289 & & 7.22 & 0.007 \\
\hline Urban & $1556(16.4)$ & & & $516(23.2)$ & & & $919(23.3)$ & & \\
\hline Rural & $2499(15.6)$ & & & $782(22.0)$ & & & $1283(21.0)$ & & \\
\hline Remarried & & 85.59 & 0.000 & & 0.00 & 0.998 & & 1.48 & 0.223 \\
\hline No & $3710(15.4)$ & & & $1046(22.1)$ & & & $1786(21.3)$ & & \\
\hline Yes & $133(32.1)$ & & & $94(22.1)$ & & & $88(19.0)$ & & \\
\hline Religion & & 52.65 & 0.000 & & 2.15 & 0.342 & & 0.56 & 0.454 \\
\hline Christians & $2035(17.7)$ & & & $1222(24.4)$ & & & $2202(21.9)$ & & \\
\hline Islam & $1991(14.4)$ & & & $6(18.8)$ & & & - & - & - \\
\hline Others & $29(17.4)$ & & & $20(29.4)$ & & & $0(0.0)$ & & \\
\hline \multicolumn{2}{|c|}{ Husband/partner's age } & 63.24 & 0.000 & & 97.88 & 0.000 & & 49.75 & 0.000 \\
\hline Less than 25 & $101(17.1)$ & & & $96(37.8)$ & & & $90(28.8)$ & & \\
\hline $25-29$ & $380(16.8)$ & & & $167(30.3)$ & & & $223(25.8)$ & & \\
\hline $30-34$ & $616(15.2)$ & & & $168(25.0)$ & & & $294(24.3)$ & & \\
\hline $35-39$ & $706(15.2)$ & & & $163(20.5)$ & & & $344(22.0)$ & & \\
\hline $40-44$ & $630(15.1)$ & & & $127(18.6)$ & & & $249(20.5)$ & & \\
\hline $45-49$ & $414(12.6)$ & & & $83(16.9)$ & & & $195(18.0)$ & & \\
\hline 50 or more & $513(11.3)$ & & & $54(12.5)$ & & & $218(16.9)$ & & \\
\hline \multicolumn{2}{|c|}{ Husband/partner's education level } & 35.79 & 0.000 & & 2.89 & 0.577 & & 11.98 & 0.017 \\
\hline No education & $1024(13.2)$ & & & $220(21.1)$ & & & $868(21.1)$ & & \\
\hline Primary & $446(13.1)$ & & & $150(24.4)$ & & & $108(18.5)$ & & \\
\hline Secondary & $1317(15.9)$ & & & $368(22.0)$ & & & $459(23.3)$ & & \\
\hline Higher & $509(13.6)$ & & & $62(21.1)$ & & & $121(18.7)$ & & \\
\hline Don't know & $64(18.9)$ & & & $58(22.9)$ & & & $57(25.2)$ & & \\
\hline \multicolumn{2}{|c|}{ Number of other wives } & 4.28 & 0.118 & & 6.95 & 0.031 & & 10.70 & 0.005 \\
\hline 1 & $2438(14.5)$ & & & $758(22.5)$ & & & $1202(22.4)$ & & \\
\hline$>1$ & $907(13.7)$ & & & $80(18.0)$ & & & $409(19.0)$ & & \\
\hline Don't know & $15(19.2)$ & & & $20(29.9)$ & & & $2(15.4)$ & & \\
\hline \multicolumn{2}{|c|}{ Husband/partner's occupation } & 5.94 & 0.015 & & 2.24 & 0.326 & & 1.79 & 0.181 \\
\hline Not working & $144(17.2)$ & & & $74(23.6)$ & & & $96(24.1)$ & & \\
\hline Working & $3216(14.2)$ & & & $782(22.0)$ & & & $1517(21.2)$ & & \\
\hline \multicolumn{2}{|c|}{$\begin{array}{l}\text { Decision maker for not using } \\
\text { contraception }\end{array}$} & 0.74 & 0.690 & & 11.11 & 0.004 & & 15.36 & 0.000 \\
\hline Mainly respondent & $1104(20.1)$ & & & $412(39.1)$ & & & $825(34.5)$ & & \\
\hline $\begin{array}{l}\text { Mainly husband, } \\
\text { partner }\end{array}$ & $585(19.5)$ & & & $102(35.4)$ & & & $272(28.3)$ & & \\
\hline Joint decision & $1258(20.2)$ & & & $215(31.3)$ & & & $361(29.9)$ & & \\
\hline
\end{tabular}




\section{Factors independently associated with unmet need for child spacing in Liberia}

The results of the chi-square test of association were presented in Table 2. In Liberia, higher proportion of women aged 15-19 years had unmet need for child spacing compared to those aged 45-49 years $(\mathrm{p}=0.000)$. Also, $25.6 \%$ of those who have attained secondary education had unmet need for child spacing $(\mathrm{p}=0.005)$. More $(26.2 \%)$ of women who were not working had unmet need for child spacing compared to those who were working (20.6\%) $(\mathrm{p}=0.000)$. Further, husbands/partners age $(\mathrm{p}=0.000)$, number of wives $(\mathrm{p}=0.031)$ and decision maker for not using contraception $(\mathrm{p}=0.004)$ were associated with unmet need for child spacing.

\section{Factors independently associated with unmet need for child spacing in Sierra Leone}

Associated factors of unmet need for child spacing were explored and the results were presented in Table 2. Higher proportion (26.2\%) of respondents aged 15-19 years had unmet need for child spacing compared to those aged 4549 years who had a lower proportion $(10 \%)(\mathrm{p}=0.000)$. Also, respondents occupation was associated with unmet need for child spacing $(\mathrm{p}=0.000)$. Similarly, wealth index (0.000), type of residence, cohabiting with husbands/partners $(\mathrm{p}=0.000)$, partners age $(\mathrm{p}=0.000)$, partners level of education $(\mathrm{p}=0.017)$, number of wives ( $\mathrm{p}=0.005)$, and decision maker for not using contraception $(\mathrm{p}=0.000)$ showed independent association with unmet need for child spacing.

\section{Factors influencing unmet need for Child spacing in Nigeria}

Logistic regression was fitted to determine the factors influencing unmet need for child spacing in Nigeria and the results was shown in Table 3. On the wealth index scale, rich women (OR: 1.14, 95\% CI: 1.02-1.28) were more likely to have unmet need for child spacing compared to poor women. Female headship of household (OR: 1.29, 95\% CI: 1.09-1.54) was associated with the likelihood of unmet need for child spacing relative to male headship of household. Also, women whose partners' age was 30-34 years (OR: $0.61,95 \%$ CI: 0.47-0.79) were 1.6 times less likely to have unmet need for child spacing compared to those aged le $<25$. Similarly, respondents whose partners' were working (OR: 0.80, 95\% CI: 0.66-0.98) were less likely to have unmet need for child spacing. In the same vein, women who having $>2$ children aged $\leq 5$ years $(\mathrm{OR}$ : 2.38, 95\% CI: $2.05-2.75$ ) were more likely to have unmet need for child spacing compared to those having $\leq 2$.

Table 3: Factors influencing unmet need for child spacing in Nigeria, Liberia and Sierra Leone.

\begin{tabular}{|c|c|c|c|c|c|c|c|c|c|c|c|c|}
\hline \multirow{3}{*}{ Variables } & \multicolumn{4}{|c|}{ Model I } & \multicolumn{4}{|c|}{ Model II } & \multirow{2}{*}{\multicolumn{2}{|c|}{ Model II }} & \multirow{2}{*}{\multicolumn{2}{|c|}{$95 \% \mathrm{CI}$}} \\
\hline & & & \multicolumn{2}{|c|}{$95 \% \mathrm{CI}$} & & & \multicolumn{2}{|c|}{$95 \% \mathrm{CI}$} & & & & \\
\hline & OR & $\mathbf{P}$ & $\mathbf{L}$ & $\mathbf{U}$ & OR & $\mathbf{P}$ & $\mathbf{L}$ & $\mathbf{U}$ & OR & $\mathbf{P}$ & $\mathbf{L}$ & $\mathbf{U}$ \\
\hline \multicolumn{13}{|c|}{ Age in 5-year groups } \\
\hline $15-19$ & \multicolumn{12}{|c|}{ Reference } \\
\hline $20-24$ & 1.33 & 0.002 & 1.11 & 1.60 & 1.12 & 0.626 & 0.72 & 1.73 & 1.14 & 0.370 & 0.86 & 1.52 \\
\hline $25-29$ & 1.32 & 0.005 & 1.09 & 1.61 & 1.22 & 0.402 & 0.76 & 1.96 & 1.20 & 0.230 & 0.89 & 1.62 \\
\hline $30-34$ & 1.5 & 0.000 & 1.22 & 1.84 & 0.71 & 0.179 & 0.43 & 1.17 & 1.06 & 0.710 & 0.77 & 1.47 \\
\hline $35-39$ & 1.32 & 0.014 & 1.06 & 1.65 & 0.55 & 0.026 & 0.33 & 0.93 & 0.93 & 0.650 & 0.67 & 1.29 \\
\hline $40-44$ & 0.98 & 0.902 & 0.76 & 1.27 & 0.37 & 0.001 & 0.21 & 0.67 & 0.58 & 0.010 & 0.39 & 0.87 \\
\hline $45-49$ & 1.04 & 0.821 & 0.76 & 1.41 & 0.26 & 0.000 & 0.12 & 0.55 & 0.38 & 0.000 & 0.23 & 0.63 \\
\hline \multicolumn{13}{|c|}{ Highest educational level } \\
\hline No education & \multicolumn{12}{|c|}{ Reference } \\
\hline Primary & 1.17 & 0.020 & 1.02 & 1.33 & 1.32 & 0.030 & 1.03 & 1.69 & - & - & - & - \\
\hline Secondary & 1.22 & 0.003 & 1.07 & 1.40 & 1.63 & 0.001 & 1.23 & 2.15 & - & - & - & - \\
\hline Higher & 0.91 & 0.343 & 0.74 & 1.11 & 0.58 & 0.157 & 0.27 & 1.24 & - & - & - & - \\
\hline \multicolumn{13}{|c|}{ Respondent currently working } \\
\hline \multicolumn{13}{|l|}{ No } \\
\hline Yes & - & - & - & - & 1.13 & 0.267 & 0.91 & 1.40 & 1.05 & 0.580 & 0.89 & 1.24 \\
\hline \multicolumn{13}{|c|}{ Number of children $\geq 5$} \\
\hline$\leq 2$ & \multicolumn{12}{|c|}{ Reference } \\
\hline$>2$ & 2.38 & 0.000 & 2.05 & 2.75 & 1.68 & 0.000 & 1.29 & 2.20 & 1.85 & 0.000 & 1.48 & 2.31 \\
\hline \multicolumn{13}{|l|}{ Wealth index } \\
\hline Poor & \multicolumn{4}{|c|}{ Reference } & - & - & - & - & & & & \\
\hline Average & 1.11 & 0.064 & 0.99 & 1.24 & - & - & - & - & 1.02 & 0.840 & 0.86 & 1.21 \\
\hline Rich & 1.14 & 0.021 & 1.02 & 1.28 & - & - & - & - & 1.07 & 0.590 & 0.83 & 1.38 \\
\hline \multicolumn{13}{|c|}{ Sex of household head } \\
\hline Male & \multicolumn{12}{|c|}{ Reference } \\
\hline Female & 1.29 & 0.004 & 1.09 & 1.54 & - & - & - & - & 0.88 & 0.180 & 0.73 & 1.06 \\
\hline
\end{tabular}




\begin{tabular}{|c|c|c|c|c|c|c|c|c|c|c|c|c|}
\hline \multirow{3}{*}{ Variables } & \multicolumn{4}{|c|}{ Model I } & \multicolumn{4}{|c|}{ Model II } & \multicolumn{4}{|c|}{ Model II } \\
\hline & \multirow[b]{2}{*}{ OR } & \multirow[b]{2}{*}{$\mathbf{P}$} & \multicolumn{2}{|c|}{$95 \% \mathrm{CI}$} & & \multicolumn{2}{|c|}{$95 \% \mathrm{CI}$} & \multicolumn{2}{|c|}{ inouer in } & \multicolumn{2}{|c|}{$95 \% \mathrm{CI}$} \\
\hline & & & L & $\mathbf{U}$ & OR & $\mathbf{P}$ & L & $\mathbf{U}$ & OR & $\mathbf{P}$ & L & $\mathbf{U}$ \\
\hline \multicolumn{13}{|c|}{ Type of place of residence } \\
\hline Urban & - & - & - & - & - & - & - & - & & & & \\
\hline Rural & - & - & - & - & - & - & - & - & 0.74 & 0.010 & 0.59 & 0.94 \\
\hline \multicolumn{13}{|l|}{ Remarriage } \\
\hline No & \multicolumn{12}{|c|}{ Reference } \\
\hline Remarriage & 0.85 & 0.589 & 0.47 & 1.54 & - & - & - & - & - & - & - & - \\
\hline \multicolumn{13}{|l|}{ Religion } \\
\hline Christians & \multicolumn{12}{|c|}{ Reference } \\
\hline Islam & 1.03 & 0.596 & 0.93 & 1.14 & - & - & - & - & - & - & - & - \\
\hline Others & 0.9 & 0.676 & 0.56 & 1.46 & - & - & - & - & - & - & - & - \\
\hline \multicolumn{13}{|l|}{ Husband/partner's age } \\
\hline Less than 25 & \multicolumn{12}{|c|}{ Reference } \\
\hline $25-29$ & 0.78 & 0.058 & 0.60 & 1.01 & 0.76 & 0.251 & 0.48 & 1.21 & 0.89 & 0.530 & 0.63 & 1.27 \\
\hline $30-34$ & 0.61 & 0.000 & 0.47 & 0.79 & 0.80 & 0.356 & 0.49 & 1.29 & 0.80 & 0.220 & 0.56 & 1.14 \\
\hline $35-39$ & 0.55 & 0.000 & 0.42 & 0.72 & 0.73 & 0.225 & 0.45 & 1.21 & 0.78 & 0.190 & 0.54 & 1.13 \\
\hline $40-44$ & 0.58 & 0.000 & 0.44 & 0.76 & 0.83 & 0.481 & 0.48 & 1.41 & 0.75 & 0.150 & 0.51 & 1.11 \\
\hline $45-49$ & 0.52 & 0.000 & 0.39 & 0.69 & 0.97 & 0.915 & 0.55 & 1.72 & 0.77 & 0.200 & 0.52 & 1.15 \\
\hline 50 or more & 0.53 & 0.000 & 0.40 & 0.71 & 0.85 & 0.599 & 0.45 & 1.58 & 0.80 & 0.270 & 0.54 & 1.19 \\
\hline \multicolumn{13}{|l|}{ Number of other wives } \\
\hline \multicolumn{13}{|l|}{1} \\
\hline$>1$ & & & & & 0.76 & 0.106 & 0.55 & 1.06 & 0.81 & 0.010 & 0.69 & 0.95 \\
\hline Don’t know & & & & & 1.54 & 0.236 & 0.75 & 3.17 & 0.52 & 0.430 & 0.10 & 2.64 \\
\hline \multicolumn{13}{|c|}{ Husband/partner's education level } \\
\hline No education & \multicolumn{12}{|c|}{ Reference } \\
\hline Primary & 0.9 & 0.158 & 0.79 & 1.04 & 1.20 & 0.248 & 0.88 & 1.63 & 0.89 & 0.380 & 0.69 & 1.15 \\
\hline Secondary & 1 & 0.967 & 0.88 & 1.14 & 1.10 & 0.459 & 0.85 & 1.43 & 1.08 & 0.360 & 0.91 & 1.28 \\
\hline Higher & 0.95 & 0.498 & 0.81 & 1.11 & 1.37 & 0.172 & 0.87 & 2.16 & 0.82 & 0.140 & 0.62 & 1.07 \\
\hline Don't know & 1.43 & 0.017 & 1.06 & 1.91 & 1.13 & 0.559 & 0.75 & 1.72 & 1.18 & 0.410 & 0.80 & 1.72 \\
\hline \multicolumn{13}{|c|}{ Husband/partner's occupation } \\
\hline Not working & \multicolumn{12}{|c|}{ Reference } \\
\hline Working & 0.8 & 0.027 & 0.66 & 0.98 & - & - & - & - & - & - & - & - \\
\hline Decision maker for not & ing co & tracept & & & & & & & & & & \\
\hline Mainly respondent & - & - & - & - & - & - & - & - & & & & \\
\hline Mainly husband, partner & - & - & - & - & - & - & - & - & 0.71 & 0.000 & 0.60 & 0.84 \\
\hline Joint decision & - & - & - & - & - & - & - & - & 0.79 & 0.000 & 0.67 & 0.92 \\
\hline
\end{tabular}

OR: Odd ratio, P: P value L: Lower limit of confidence interval, U: Upper limit of confidence interval

\section{Factors influencing unmet need for child spacing in Liberia}

Factors that were identified as influencing factors of unmet need for child spacing in Liberia were presented in Table 3. The likelihood of unmet need for child spacing decreased as age increased. For instance, women aged 3539 years (OR: $0.55,95 \%$ CI: $0.33-0.93), 40-44$ years $(\mathrm{OR}$ : $0.37,95 \%$ CI: $0.21-0.67$ ), and $45-49$ years (OR: $0.26,95 \%$ CI: 0.0.12-0.55) were less likely to have unmet need for child spacing. Also, respondents with primary education (OR: 1.32, 95\% CI: 1.03-1.69) and secondary education (OR: 1.63, 95\% CI: 1.23-2.15) were more likely to have unmet need for child spacing compared to their uneducated counterparts. In similar vein, women who are having $>2$ children aged $\leq 5$ years (OR: $1.68,95 \%$ CI: 1.29-2.20) were more likely to have unmet need for child spacing.

\section{Factors influencing unmet need for child spacing in Sierra Leone}

As presented in Table 3. Respondents aged 40-44 years (OR: $0.58,95 \%$ CI: $0.39-0.87$ ) and $45-49$ years (OR: 0.38 , 95\% CI: 0.23-0.63) were less likely to have unmet need for child spacing compared to women aged 15-19 years. Having more than two number of children (OR: 1.85, 95\% CI: 1.48-2.31) was associated with the likelihood of unmet need for child spacing compared to having $\leq 2$ number of children. Surprisingly in Sierra Leone, we found out that women living in the rural area (OR: $0.74,95 \%$ CI: $0.59-$ 0.94) were less likely to have unmet need for child spacing. In furtherance, women whom their husbands have more than one wife (OR: 0.81, 95\% CI: 0.69- 0.95), those whom their husbands/partners mainly took decision for not using contraception (OR: $0.71,95 \% \mathrm{CI}: 0.60-0.95$ ) and those 
who jointly took decision for not taking contraception with their husbands (OR: 0.79, 95\% CI: 0.67-0.92) were less likely to have unmet need for child spacing.

\section{DISCUSSION}

In this study, we determined the prevalence of unmet need for child spacing in the selected three sub-Saharan countries (Nigeria, Liberia and Sierra Leone) that were experiencing high maternal mortality. We as well examine the influence of demographic characteristics, socioeconomic, and partner's profile on unmet need for child spacing among women of reproductive age in Nigeria, Liberia and Sierra Leone. We found a high prevalence of unmet need for child spacing among the study population. The prevalence of unmet need for spacing in Liberia and Sierra Leone were a bit close but, both staggered from that of Nigeria. In other words, about one out of every six in Nigeria, close to a quarter in Liberia and two out of every nine in Sierra Leone had unmet need for child spacing. There were similarities in the results found in Liberia and Sierra Leone, and that of another study carried out in Cameroon. ${ }^{2}$ But higher than the result found in Mexico. ${ }^{15}$ These diverse prevalence could be as a result of varying cultural believe about reproduction in these study settings and level of efforts invested into the uptake of contraception. Risk factors of unmet need for child spacing that were found across the three countries were younger age (below 40 years), primary and secondary education. Also, female headship of households and partner's occupation status were influencing factors of unmet need for child spacing in Nigeria. These influencing factors are not peculiar to this study population alone, studies in Zambia and Ghana also reported similar findings. ${ }^{2}$ These identified factors could be narrowed down to unfavourable decision of uptake of FP as a result of immaturity, ignorance poverty. The justification for the unhealthy child spacing among the uneducated and those who are not working is not farfetched as an English proverb said "children are poor man's riches" and that more children is the consolation of a poor man. ${ }^{16}$ It will be dangerous to assume that academics improved uptake of contraception, i.e. specific education on uses, side effects and benefits of contraception should be employed in reducing unmet need for contraception.

However, it's quite not expected that partners' who are not working would have a high unmet need for child spacing because family planning services are provided free of charge in this study population. This could imply that there is a masking factor of the unhealthy child spacing among women whose husbands were not working.

In line with previous study, we found out that women having more under five children in the household were more likely to experience unmet need for spacing. ${ }^{17}$ Stress of parenting and contraception uses may have a hidden interactions as women having more under five children were more likely to have unmet need for child spacing. ${ }^{15}$ This could be traced to the theory of parenting stress, which is a major cause of maternal complication. ${ }^{4}$ However, more powerful study design or powerful statistical techniques could be used to further unmask and confirm the relationship between parenting stress and unmet need for contraceptive.

This study has some limitations. In this study, we used a secondary data. It was only the available data that we could analyse and work with. Some of our own specific research questions or specific information that we would like to have couldn't be asked, this means it's not specific to our needs as researchers. ${ }^{18-34}$

\section{CONCLUSION}

Unmet need for child spacing was high in the three countries, namely Nigeria (15.9\%), Liberia (22.5\%), and Sierra Leone $(21.9 \%)$. Factors that should gain enough focus in the efforts to improve FP programs so as to reduce maternal mortality and maternal complication and bring improvements to the health of women of reproductive age were identified in this study. For instance, women of lower education and advanced reproductive age should be given much attention. A novel finding in this study is that women who are going through parenting of children of five years and below should not be left in the strategic policy dialogue to curb the menace. Also, women whom their husbands/ partners were not engaged with any occupation need to be properly addressed in this matter, they need to be sufficiently enlightened about the danger of improper child spacing to their health.

\section{Recommendations}

In the battle against maternal mortality, pregnancy related mortality and complications resulting from unintended pregnancies, women with lower level of education, younger age, having $>2$ five/under five year old children in the household, as well as women who are heading their household should be given much attention in the efforts to reduce unmet need for child spacing. Also, it's well known that efforts are ongoing by non-governmental organizations and several initiatives in Nigeria, Liberia and Sierra Leone. Findings from this study should be carefully incorporated into their planning to achieve the SDG goals on improving the health of women of reproductive age.

\section{ACKNOWLEDGMENTS}

Authors are thankful to Mr Abayomi T. Olarinmoye, $\mathrm{Mr}$ Olanrewaju D. Eniade, Mrs Olariike O. Mrs Kayode, Abimbola A. Abiola, Mrs Modupe O. Onifade, Mr Sarafa O. Odewale, and Professor Bode Kayode.

\author{
Funding: No funding sources \\ Conflict of interest: None declared \\ Ethical approval: The study was approved by the \\ Institutional Ethics Committee
}




\section{REFERENCES}

1. Ahmed S, Li Q, Liu L, Tsui AO. Maternal deaths averted by contraceptive use: an analysis of 172 countries. Lancet. 2012;380(9837):111-25.

2. Almalik MM. Understanding maternal postpartum needs: a descriptive survey of current maternal health services. J Clin Nurs. 2017;26(23-24):4654-63.

3. Bahk J, Yun SC, Kim YM, Khang YH. Impact of unintended pregnancy on maternal mental health: a causal analysis using follow up data of the Panel Study on Korean Children (PSKC). BMC Pregnancy Childbirth. 2015;15(1):85.

4. Deater-Deckard K. Parenting stress. Yale University Press; 2014:220.

5. Dehingia N, Dixit A, Atmavilas Y, Chandurkar D, Singh K, Silverman J, Raj A. Unintended pregnancy and maternal health complications: cross-sectional analysis of data from rural Uttar Pradesh, India. BMC Pregnancy Childbirth. 2020;20:1-11.

6. Demographic and Health Survey. Version 20. Report on contraceptive, published by Nigeria Bureau of Statistics, Nigeria. 2017. Available from: https://dhsprogram.com. Accessed on 10 May 2021.

7. Essi MJ, Yakum MN, Mbu ER, Bhusal CK. Factors affecting unmet need of family planning among married Tharu women of Dang District, Nepal. BMC Womens Health. 2018;9312687.

8. Fagbamigbe AF, Afolabi RF, Idemudia ES. (2018). Demand and unmet needs of contraception among sexually active in-union women in Nigeria: distribution, associated characteristics, barriers, and program implications. SAGE Open. 2018;8(1).

9. Fure E. Are children poor men's riches? Scand Econom History Rev. 1983;31(3):161-77.

10. Getaneh T, Negesse A, Dessie G, Desta M, Moltot T. Predictors of unmet need for family planning in Ethiopia 2019: a systematic review and meta-analysis. Arch Public Health. 2020;78;102.

11. Guure C, Maya ET, Dery S, da-Costa VB, Alotaibi RM, Rezk HR, et al. (2019). Factors influencing unmet need for family planning among Ghanaian married/union women: a multinomial mixed effects logistic regression modelling approach. Arch Public Health. 2019;77:11.

12. National Institute of Statistics and Economic Analysis, and ICF. (2019). Republic of Benin Fifth Demographic and Health Survey in Benin (EDSB-V) 2017-2018. Cotonou, Benin: INSAE/Benin and ICF.

13. Juarez F, Gayet C, Mejia-Pailles G. Factors associated with unmet need for contraception in Mexico: evidence from the National Survey of Demographic Dynamics 2014. BMC Public Health. 2018;18(1):1-8.

14. Klaus K. (2021). Crna Gora-Country Profile: Montenegro. Nations online.org, Stle-(12 October 1992). Available from: https://www.nationsonline. org/oneworld/montenegro.htm. Accessed on 13 May 2021.

15. Mulenga JN, Bwalya BB, Mulenga MC, Mumba K. Determinants of unmet need for family planning among married women in Zambia. J Public Health Afr. 2020;11(1):1084.

16. National Population Commission- NPC, and ICF. (2019). Nigeria Demographic and Health Survey 2018- Final Report. Abuja, Nigeria: NPC and ICF. Available from: https://dhsprogram.com/ pubs/pdf/FR359/FR359.pdf. Accessed on 13 May 2021.

17. United Nation. 2011 report: World contraceptive use. United Nation. Available at: https://www.un.org/en/development/desa/population/ publications/family/contraceptive-wallchart2011.asp. Accessed on 20 July 2021.

18. Nzokirishaka A, Itua I. Determinants of unmet need for family planning among married women of reproductive age in Burundi: a cross-sectional study. Contracept Reprod Med. 2018;3(1):1-13.

19. Okigbo CC, McCarraher DR, Chen M, Gwarzo U, Vance G, Chabikuli O. Unmet need for contraception among clients of FP/HIV integrated services in Nigeria: the role of partner opposition. Afr J Reprod Health. 2014;18(2):134-43.

20. Pack AP, McCarraher DR, Chen M, Okigbo CC, Albert LM, Wambugu S. Factors associated with unmet need for modern contraception in post-conflict Liberia. Afr J Reprod Health. 2014;18(2):58-67.

21. Regan L. Addressing unmet needs in global women's health. British Medical Association. 2018. Available from: https://www.bma.org.uk/media/2117/bmawomens-global-health-report-aug-2018.pdf. Accessed on 20 July 2021.

22. Sedgh G, Ashford LS, Hussain R. Unmet need for contraception in developing countries: examining women's reasons for not using a method. New York: Guttmacher Institute; 2016.

23. Stigum H, Htay TT, Liabsuetrakul T, Moe Myint AN, Sundby J, Solomon T, et al. Unmet need for family planning and associated factors among currently married reproductive age women in Tiro Afeta District, South West Ethiopia, 2017: cross-sectional study. Int J Environ Res Public Health. 2019;19(1):170.

24. Surkan PJ, Strobino DM, Mehra S, Shamim AA, Rashid M, Wu LSF, et al. Unintended pregnancy is a risk factor for depressive symptoms among socioeconomically disadvantaged women in rural Bangladesh. BMC Pregnancy Childbirth. 2018;18(1):1-13.

25. Tsegaye D, Shuremu M, Bidira K. Practice of child spacing and its associated factors among women of child bearing age (15 to 49 years) in Illubabor zone, South West Ethiopia. Int $\mathbf{J}$ Nurs Midwife. 2017;9(7):102-8.

26. UNICEF. (2017). Maternal mortality declined by 38 per cent between 2000 and 2017. Available from: https://data.unicef.org/topic/maternal-

health/maternal-mortality/. Accessed on 10 May 2021.

27. United Nations. (2017a). Goal 11: Make Cities Inclusive, Safe, Resilient and Sustainable, Sustainable 
Development Goals. United Nation. Available at https://sdgs.un.org/goals/goal11. Accessed on 13 May 2021.

28. Utomo B, Sucahya PK, Romadlona NA, Robertson AS, Aryanty RI, Magnani RJ. The impact of family planning on maternal mortality in Indonesia: what future contribution can be expected? Popul Health Metrics. 2021;19(1):1-13.

29. World Health Organization. Report of a WHO technical consultation on birth spacing: Geneva, Switzerland 13-15 June 2005: World Health Organization. 2007. Available from: https://apps.who.int/iris/handle/10665/69855.

Accessed on 13 May 2021.

30. World Health Organization. Contraception within the context of adolescents' sexual and reproductive lives: country factsheet: Afghanistan: World Health Organization. 2021. Available from: https://apps.who.int/iris/handle/10665/339250. Accessed on 13 May 2021.

31. Worldometers, i. (2018). Nigeria Population (2018). Available at: https://www.worldometers.info/world- population/nigeria-population/. Accessed on June 6 2021.

32. Wulifan JK, Mazalale J, Kambala C, Angko W, Asante J, Kpinpuo S, et al. Prevalence and determinants of unmet need for family planning among married women in Ghana-a multinomial logistic regression analysis of the GDHS, 2014. Contracept Reprod Med. 2019;4(1):1-14.

33. Wulifan JK, Mazalale J, Kambala C, Angko W, Asante J, Kpinpuo S, et al. Prevalence and determinants of unmet need for family planning among married women in Ghana-a multinomial logistic regression analysis of the GDHS. Contracept Reprod Med. 2019;4(1):1-4.

34. Yaya S, Ghose B. Prevalence of unmet need for contraception and its association with unwanted pregnancy among married women in Angola. PloS One. 2019;13(12).

Cite this article as: Olarinmoye AT, Eniade OD, Kayode OO, Abiola AA, Onifade MO, Odewale SO, et al. Influencing factors of unmet needs for child spacing among selected countries experiencing high maternal-mortality in sub-Saharan Africa. Int J Reprod Contracept Obstet Gynecol 2021;10:4361-71. 